\title{
17 \\ Conformance testing of ATM Adaptation Layer protocol
}

\author{
Sangjo Yoo *, Leslie Collica ${ }^{* *}$, and Myungchul Kim * \\ *Korea Telecom Research and Development Group \\ Sochogu Unyundong 17, Seoul, 137-795, Korea \\ \{sjyoo,mckim\}@sava.kotel.co.kr \\ ** National Institute of Standards and Technology \\ Building 223/ Room B364, Gaithersburg, MD 20899, USA \\ collica@isdn.ncsl.nist.gov
}

\begin{abstract}
This paper presents an appropriate testing methodology to check the conformity of an implementation following ATM Adaptation Layer protocol in ATM/B-ISDN communication systems. In this paper, we analyze restrictions of the AAL Type 5 protocol to develop Abstract Test Suites and a test system, and we compare test coverage of applicable test methods to choose the best test method for AAL Type 5 Common Part protocol testing. We decide to apply Distributed Test Method for testing of the protocol because of its larger test coverage. To synchronize the execution of test cases between Upper Tester and Lower Tester in distributed testing environment, we propose a simple and efficient timing synchronization mechanism. To illustrate the effectiveness of our test structure, we give an example of test cases described in Tree and Tabular Combined Notation using the proposed timing synchronization mechanism.
\end{abstract}

\section{Keywords}

Conformance Testing, ATM Adaptation Layer, Distributed Testing, TTCN, Upper Tester

\section{INTRODUCTION}

Asynchronous Transfer Mode (ATM) and Broadband Integrated Services Digital Network (B-ISDN) are rapidly becoming acknowledged as a base technology for the next generation of global communications supporting a variety of applications. ATM/B-ISDN protocols have very complex structures compared with narrowband ISDN or packet networks.

Of the ATM/B-ISDN protocols, ATM Adaptation Layer (AAL) (ITU-T COM SVIII-R 116, 1992)is used to enhance the services provided by ATM layer to support the functions required by the layer above. Therefore, the functions performed in the AAL depend upon the higher layer requirements. 
Especially, the AAL Type 5 protocol (ITU-T TD-XVIII/10, 1993) is defined not only for user data transfer in the user plane, but also for signalling in the control plane. To ensure that an implementation conforms to its standards or specifications and then to increase the probability of interoperability between ATM/B-ISDN products from multiple venders, protocol conformance testing in telecommunication has become an important area in the latest years. In order to assure the sufficient test coverage and to perform the conformance testing in accordance with the test requirements, first of all, we should decide the most appropriate test method and test configuration. In this paper, we present an appropriate test methodology using the proposed timing synchronization mechanism for the AAL Type 5 protocol conformance testing.

In Section 2, we analyze the restrictions of AAL Type 5 protocol in developing Abstract Test Suites (ATSs) and a test system for conformance testing. In Section 3, we compare test coverage for test methods, and we list advantages and disadvantages of each method. In Section 4, we show applicable test configuration for AAL Type 5 Common Part protocol conformance testing. In Section 5, we propose a simple and efficient timing synchronization procedure in Distributed Test Method (DTM). The procedure has no additional requirements or management protocols for synchronizing between Upper Tester (UT) and Lower Tester (LT). In Section 6, we show the requirement and implementation structure of the UT. In Section 7, we give an example of test cases, described in Tree and Tabular Combined Notation (TTCN) (ISO/IEC 9646-3, 1993), using the proposed timing synchronization mechanism for AAL Type 5 Common Part protocol. Finally, in Section 8, we conclude this paper.

\section{RESTRICTIONS OF AAL PROTOCOL FOR CONFORMANCE TESTING}

In order to develop test suites for conformance testing, we should consider test requirements that specify which protocol characteristics should be tested. Once we have defined test requirements including PICS (Protocol Implementation Conformance Statement) proforma, we need to decide what kind of test methods can be used to specify the abstract test suites. Then we can specify test scenarios describing test behaviour between tester and Implementation Under Test (IUT) as an ATS, and we can implement a test system based on the test scenarios. Before we decide a test method, we should carefully review all the restrictions in the protocol under test. In this section, we analyze the restrictions on conformance testing of AAL Type 5 protocol. follows:

Major factors in AAL Type 5 specification [2] that restrict conformance testing are listed as

- various profiles,

- no Service Access Point (SAP) between sublayers,

- simple Protocol Data Unit (PDU) transfer procedure, and

- absence of description for control and management planes.

\subsection{Various Profiles}

As shown in Figure 1 [2], the AAL Type 5 protocol consists of CPAAL 5 (Common Part AAL Type 5), that is independent of AAL service user, and Service Specific Convergence Sublayer (SSCS), that is dependent on the characteristics of AAL service user. This means that a variety of AAL Type 5 profiles may exist according to the kind of SSCS protocols (including a case that SSCS is null) that support specific AAL user services or groups of services. Examples of possible AAL Type 5 structures are shown as follows: 
- AAL Type 5 structure consisting of only CPAAL 5 (i.e., SSCS is null),

- Signalling AAL consisting of CPAAL 5, Service Specific Connection Oriented Protocol (SSCOP), and Service Specific Coordination Function (SSCF). SSCOP provides assured data delivery between AAL connection endpoints, and SSCF performs a coordination function between the service required by user of signalling layer 3 (Q.2931) and the service provided by SSCOP, and

- Other AAL Type 5 structures depending on SSCS protocol (e.g., FR-SSCS over CPAAL 5 for Frame Relay service).

The fact that we have these various profile structures means that several kinds of test suites are necessary. If we want to use UT for conformance testing on AAL Type 5 protocol, we have to define and implement UT functions according to the profile of AAL Type 5.

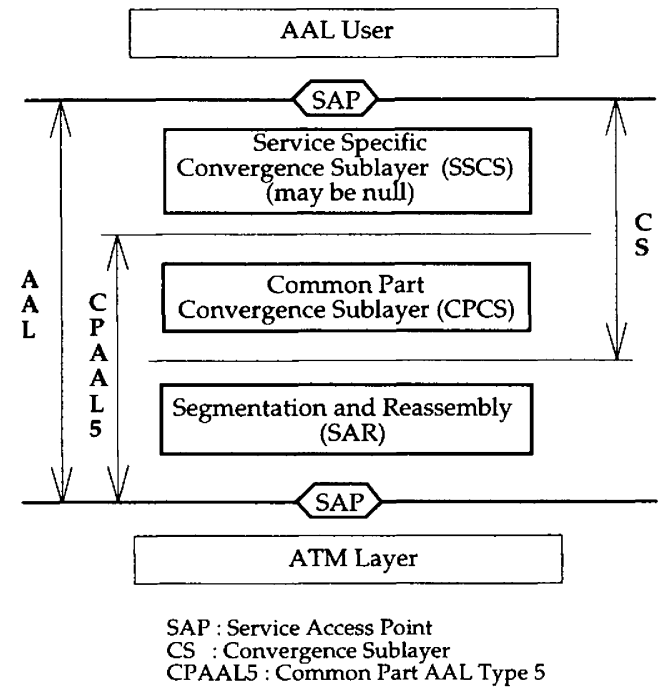

Figure 1 Structure of AAL Type 5

\subsection{No SAP Between Sublayers of AAL Type 5}

AAL Type 5 protocol is subdivided into multiple sublayers as shown in Figure 1. According to ATM Adaptation Layer Type 5 specification in ITU-T I.363 draft recommendation [2], there exist SAPs between the AAL and the ATM layer, and between the AAL and the AAL user. However there exist no SAPs between sublayers of AAL Type 5. This means that exchanges of parameters (e.g., AAL SDU (Service Data Unit), loss priority, and congestion indication) between sublayers can be implemented by internal interface method of each implementation.

In protocol conformance testing, the fact that there exist no SAPs between sublayers implies that it is very difficult for UT and LT of a test system to access interfaces between sublayers directly. Thus, this gives us restrictions in choosing a test method and implementing a test system. 


\subsection{Simple PDU Transfer Procedure of CPAAL 5}

CPAAL 5 protocol has a very simple protocol behaviour and does not have any PDU (Protocol Data Unit) exchanges between the peer entities for connection establishment or flow control to transfer CPCS-SDUs (Common Part Convergence Sublayer - Service Data Units). Both CPCS and SAR (Segmentation And Reassembly) have only one state (IDLE state). Thus there exists no state transition except a self transition. However, the simple procedure of the protocol gives us difficulty to observe the behaviour of the protocol entity from the outside on the viewpoint of black box testing.

CPAAL 5 does not send any CPCS and SAR PDUs and does not have state transitions when it receives SAR-PDUs and CPCS-PDUs from the peer entity. Thus, testing of the CPAAL 5 protocol for the error handling and appropriate parameter passing at the receiver side has to be dependent on the direct observation of the service primitives from the CPAAL 5 upper interface or of the behaviour of the CPAAL 5 user layer entity.

In a strict sense, SAR does not have its Protocol Control Information (PCI) and error handling procedures. Therefore, if CPCS entity receives an errored CPCS-PDU, it is actually impossible to know whether the error is caused by the fault on SAR or the fault on CPCS entities. This means that when we perform CPAAL 5 conformance testing, separate single-sublayer testing can not satisfy the test requirements including error localization.

\subsection{Absence of Descriptions for Control and Management Plane}

The descriptions for the control and management plane of CPAAL 5 are not included in ITU-T draft recommendation I.363 [2] and they remain for further study. Thus the possible test coverage for CPAAL 5 is restricted to the user plane specification, and additional study on the control and management plane is required in future.

\section{TEST COVERAGE ANALYSIS}

To choose the most efficient test method for AAL Type 5 protocol conformance testing, in this section, we compare the test coverage of applicable test methods. In fact, test coverage is largely affected from the test method itself. For more reliable and precise testing, we should use a test method that has larger test coverage than those of others.

In this section, we analyze test methods in the viewpoint of advantages and disadvantages of each method for AAL Type 5 conformance testing. And we present a result on comparing test coverage of each test method.

\subsection{Comparison of Test Methods for AAL Type 5 Conformance Testing}

For protocol conformance testing, four abstract test methods have been standardized by ISO and ITU$\mathrm{T}$ as ISO/IEC IS 9646 part 1 (ISO/IEC 9646-1, 1993) and ITU-T X.290 (ITU-T X.290, 1992) respectively. These methods are Local Test Method, Distributed Test Method, Coordinated Test Method, and Remote Test Method.

Among four standardized test methods, Local Test Method is the simplest form. The tester in Local Test Method surrounds an IUT, and it is able to control and observe the interfaces of a layer above and below. However this test method is only a conceptual architecture because most implementations do not provide both interfaces externally. For most commercial network equipments, AAL Type 5 protocol is implemented as an embedded layer between ATM layer and AAL user. Therefore the application of 
Local Test Method to AAL Type 5 protocol conformance testing is not feasible. In case of Coordinated Test Method, a standardized Test Management Protocol (TMP) is required for coordinating between UT and LT. But the TMP requires extra cost for developing the protocol.

Because of above reasons, Local Test Method and Coordinated Test Method are not considered in AAL testing. Remote Test Method and Distributed Test Method are candidates as applicable test methods for AAL Type 5 protocol testing.

\section{1) Remote Test Method}

Remote Test Method is the simplest one to describe a test specification and to implement a test system. The advantages and disadvantages of Remote Test Method for AAL Type 5 testing are as follows:

\section{Advantages}

- It is simple to design and to implement the test suite and the test system because we need to consider only LT functions to observe and control IUT through the lower interface of the LT.

- This test method makes no special demands on SUT (System Under Test) for testing (i.e., Remote Test Method makes no assumptions about the internal design of SUT or IUT, and treats SUT as a black box).

\section{Disadvantages}

- The control and observation of IUT is performed only through the lower interface of LT. Therefore there are limitations on test coverage. Especially, in case of the common part of AAL Type 5, a main function at receiver mode is to pass correct CPCS user data and parameters to the CPCS user after error handling. And, as presented in Section 2.3, CPAAL 5 protocol does not have any PDU exchanges between the peer entities for connection establishment or flow control. Therefore, if AAL Type 5 consists of CPAAL 5 only, it is impossible to test the receiver mode of IUT using Remote Test Method which can not control and observe ASPs (Abstract Service Primitives) from IUT upper interface.

- There are limitations on AAL user commands for testing. In AAL Type 5 sender mode testing, tests for various AAL user data conditions (e.g., testing under different AALSDU length sizes or parameter values) may be needed. But in case of Remote Test Method, it is difficult to control AAL user data conditions that we want to set.

- Manual operations may be required because there are no demands on SUT to implement UT functions and to perform the test coordination between UT and LT.

\section{2) Distributed Test Method}

Distributed Test Method uses PCOs (Point of Control and Observations) at LT and UT, thus we are able to control and observe IUT through PCOs. The advantages and disadvantages of Distributed Test Method are as follows:

\section{$\underline{\text { Advantages }}$}

- This test method has a larger test coverage than that of Remote Test Method because we can control and observe IUT using LT and UT. 
- The test coordination procedures are made more rigorously in a sense that it can include control and observation instructions related directly to ASPs across AAL and AAL user layer boundary.

\section{Disadvantages}

- The direct access to the upper boundary is needed to implement UT in SUT.

- Extra cost is needed to design a complex test suite and to implement special requirements for UT.

\subsection{Comparison of Test Coverage}

AAL Type 5 Common Part Protocol is independent of AAL service user, and it is used as a common protocol for all AAL Type 5 profiles. Before we develop an abstract test suite for CPAAL 5 conformance testing, we compared the test coverage of different types of test methods.

Much related work has been done in deriving test sequence directly from Finite State Machine (FSM) and formal specifications described in formal description techniques such as SDL, ESTELLE, and LOTOS (Krishan Sabnani and Anton Dahbura, 1988)(Tsun S. Chow, 1978)(G. Gonenc, 1970)(Ana R, Cavalli, Jean-Philippe Favreau, and Marc Phalippou, 1994). However, AAL5 Common Part (CPAAL5) protocol specified in ITU-T I.363 does not have state transitions listed as one of the testing restrictions in Section 2.3. Therefore, it is difficult to generate test sequences automatically for all instances of specification.

For testing AAL5 Common Part, the ATM Forum Test Working Group developed test purposes (Nouni, 1995) manually. There are thirty four test purposes, with fourteen test purposes in PDU Format, eight in Sender Mode, and twelve in Receiver Mode. These test purposes are considered to have covered all major functions of the protocol specification. Because of absence of descriptions for the control and management plane (see Section 2.4), test purposes for management and control functions have not been defined. For comparing test coverage for each test method to be used in developing ATS, the test purpose document (Nouni, 1995) is used for the reference target. Test coverage is presented in terms of percentage of realizable tests in the test purposes document.

\section{1) Chosen Test Methods}

This section presents differences between two kinds of Distributed Test Method and assumptions in using Remote Single Layer Test Method. We assume that SUT having CPAAL 5 protocol supports direct access to the upper boundary of CPAAL 5 to implement an UT.

\section{Type 1 of Distributed Test Method (D1)}

- UT can control and observe only CPCS-SDU parameter among CPCS-primitives.

- UT has minimal functions required for conformance testing. Therefore it is easy to implement UT functions in SUT. This kind of test method can be used more generally in testing AAL Type 5 because the only UT functions required are normal sending and receiving of AAL User Data.

\section{Type 2 of Distributed Test Method (D2)}

- UT can control and observe all parameters of CPCS-primitives in addition to D1 capabilities listed above. 


\section{Remote Single Layer Test Method (R)}

- It is assumed that UT supports implicit sending of CPCS-PDUs for testing (if IUT can not support this sending for testing, test coverage will be decreased).

\section{2) Comparison of Test Coverage}

A summary of test coverage comparison for the three applicable test methods is presented in Table 1 . While all test purposes are testable in Type 2 of Distributed Test Method (D2), only 47\% (16 test purposes) of test purposes (34 test purposes) are testable in Remote Test Method, and 82\% (28 test purposes) are testable in Type 1 of Distributed Test Method (D1). Furthermore the test coverage for Remote Test Method may be decreased if testing client can not answer some of PIXIT (Protocol Implementation eXtra Information) questions (for example, can the content of CPCS-SDU (payload) be known by the tester/test operator prior to testing?).

The $18 \%$ difference between D1 and D2 test methods comes from the fact that UT in D1 can not handle CPCS-UU, Congestion Indication, and Loss Priority parameters.

It is impossible to test the receiver mode of IUT using Remote Test Method because it can not control and observe the abstract service primitives of IUT upper interface. Only by observing the lower interface of IUT in Remote Test Method, a test system (or test operator) can not know whether IUT discards CPCS-PDUs and parameters or delivers them to CPCS because CPAAL 5 protocol does not send any PDUs to respond upon reception of SAR-PDUs or CPCS-PDUs from the peer entity, as shown in Section 2.3.

Table 1 Summary of test coverage comparison

\begin{tabular}{|c|c|c|c|c|c|c|}
\hline & \multicolumn{2}{|c|}{ Distributed Test (D1) } & \multicolumn{2}{c|}{ Distributed Test (D2) } & \multicolumn{2}{c|}{ Remote Test (R) } \\
\cline { 2 - 7 } & Testable & $\begin{array}{c}\text { Non- } \\
\text { testable }\end{array}$ & Testable & $\begin{array}{c}\text { Non- } \\
\text { testable }\end{array}$ & Testable & $\begin{array}{c}\text { Non- } \\
\text { testable }\end{array}$ \\
\hline \hline PDU Format & 13 & 1 & 14 & 0 & 10 & 4 \\
\hline Sender Mode & 6 & 2 & 8 & 0 & 6 & 2 \\
\hline Receiver Mode & 9 & 3 & 12 & 0 & 0 & 12 \\
\hline \hline $\begin{array}{c}\text { Total number of } \\
\text { Test Purposes (\%) }\end{array}$ & $28(82 \%)$ & $6(18 \%)$ & $34(100 \%)$ & $0(0 \%)$ & $16 *(47 \%)$ & $18(53 \%)$ \\
\hline
\end{tabular}

* Note : does not require UT function.

\section{TESTING CONFIGURATION FOR CONFORMANCE TESTING OF AALTYPE 5 COMMON PART}

As explained in Section 2.2, there are a number of AAL Type 5 profile structures that consist of different sublayers depending on AAL user characteristics. In this section, we present an appropriate test configuration for AAL Type 5 profile consisting of CPAAL5 only.

If AAL Type 5 consists of CPAAL 5 only, the profile structure of AAL Type 5 has SAR and CPCS protocols only. In this case, as analyzed in Section 3, Remote Test Method can not cover receiver mode 
tests for AAL Type 5 protocol. To test the receiver mode of IUT, a tester needs UT function. Even in the sender mode testing, test requirements for various AAL user conditions may be needed. However, if we use Remote Test Method, it is difficult to control AAL user commands that stimulate a variety of AAL user conditions. For these reasons, we chose Distributed Layer Test Method that allows access to the lower and upper boundaries of IUT as shown in Figure 2.

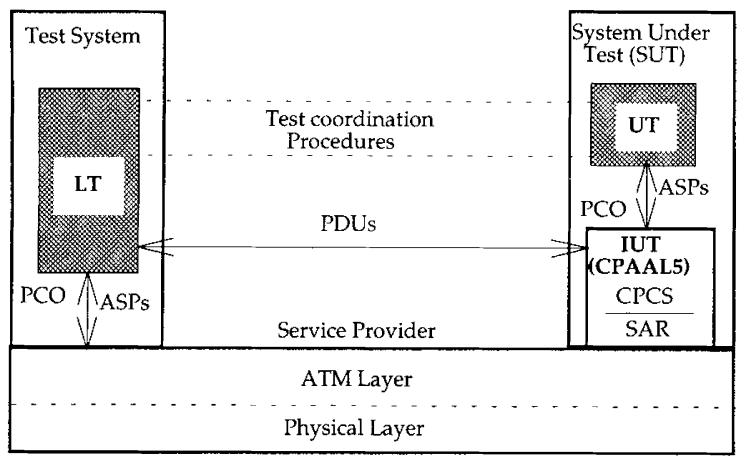

Figure 2 Test configuration for conformance testing of CPAAL 5 protocol

\section{TIMING SYNCHRONIZATION AND TEST COORDINATION BETWEEN LT AND UT FOR CPAAL5 TESTING}

Timing synchronization and test coordination between LT and UT are very important issues in the design and implementation of LT and UT. Especially in case of Distributed Test Method, synchronization and coordination procedure between LT and UT are practical issues, because there is no standardized test management protocol as in Coordinated Test Method. For synchronization and coordination, we can use the ferry principle (Zeng H.X, Chanson S.T., and Smith B.R., 1989)(Marc F. Witteman and Ronald C. van Wuijtswinkel, 1993) or define a user-defined protocol for testing. However, both of these approaches require extra costs to develop a test suite and a test system.

Fortunately, CPAAL 5 protocol is a simple protocol, and there is no requirement for complex protocol timers. Therefore, the synchronization problem becomes a coordination problem between LT and UT. In this section, a simple and efficient timing synchronization procedure is presented without any additional requirements or protocol for timing synchronization between UT and LT.

\subsection{Timers}

For conformance testing of AAL Type 5 Common Part protocol using Distributed Test Method, we have defined three timers to achieve timing synchronization and coordination between UT and LT during testing.

\section{1) Preamble Timer : T_PRE}

This timer is used by LT to make a verdict as an "INCONCLUSIVE" when the LT does not receive 
the expected response from IUT before this timer expires after the LT sent an ATM_DATA.request primitive ${ }^{1}$ to notify a test case starting in a preamble.

\section{2) Sender Mode (of the IUT) Timer : T_SEND}

This timer is used by LT to define a period of time which the LT has to wait to guarantee receiving all ATM_DATA.indication primitives that were sent by UT as AAL user data from IUT.

\section{3) Receiver Mode (of the IUT) Timer : T_REC}

This timer is used by UT to define the time that UT has to wait to guarantee receiving the expected AAL user data as an AAL_UNITDATA.indication primitive that was sent by LT as pre-defined ATM_DATA.request primitives.

To determine the timer durations for three synchronization timers listed above, we have defined several parameters (e.g., Tr, Td1, and Ts which were presented in Table 2, 3, and 4) as PIXIT (Protocol Implementation eXtra Information for Testing) proforma questions. The values for these parameters can be determined experimentally during the testing preparation step by the test operator and the test client.

\subsection{Timing Synchronization and Test Coordination Method}

Proposed timing synchronization and test coordination procedure between LT and UT starts with sending an ATM primitive that includes the start message and the test case identification to be executed by LT. When UT receives the start message, it sends an acknowledge message to the IUT. Immediately after sending and receiving the acknowledge message, the UT and LT perform predefined test procedure using the proposed timers(Sangjo Yoo and Leslie Collica, 1995).

\section{1) Preamble Timer : T_PRE}

For execution of a test case, we need a preamble procedure so that LT gives UT the test case identification to be executed. In the preamble, "Start (test_case_id)" ATM_DATA.request primitive and "Start (test_case_id)" AAL_UNITDATA.indication primitive are used to notify the starting of a specific test case "test_case_id" to the UT. "Ack (test_case_id)" ATM_DATA.indication primitive and "Ack (test_case_id)" AAL_UNITDATA.request primitive are also used for starting point (time) of other timers such as T_SEND and T_REC by LT and UT, respectively. Figure 3 shows an example of T_PRE timer operation. The parameters for the preamble timer operation are shown in Table 2.

After sending "Start" ATM primitive, LT starts T_PRE timer. If LT receives an "Ack" ATM primitive from IUT, LT stops timer T_PRE, then executes the test body of the LT part. On receipt of "Start" AAL primitive containing the test case start message and the test case number, UT sends "Ack" AAL primitive to IUT, and then executes the test body of UT part. When timer T_PRE expires prior to receipt of an "Ack" ATM primitive from IUT, LT stops the test case and assign a verdict as an "INCONCLUSIVE".

\footnotetext{
1 An ATM_DATA.request or ATM_DATA.indication primitive can be implemented as a corresponding ATM cell which has correct primitive parameters (i.e., Cell Loss Priority, Congestion Indication, and ATM Payload) in the ATM cell header and payload.
} 


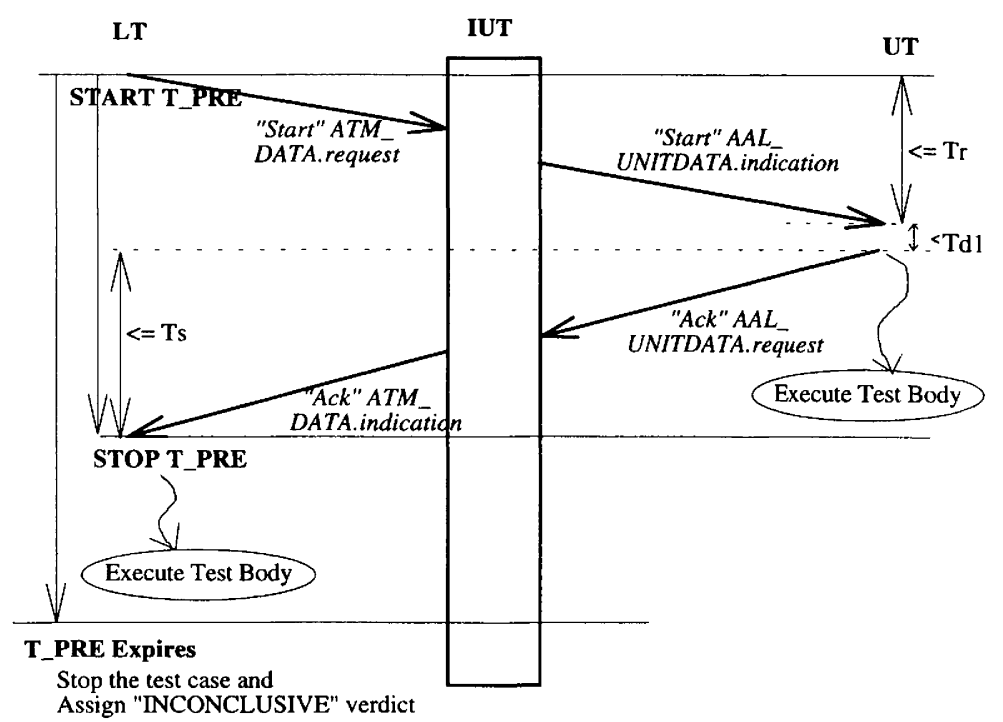

Figure 3 Preamble T_PRE timer operation

Table 2 Parameters for preamble timer operation

\begin{tabular}{|c|l|}
\hline Timer/Parameter Name & \multicolumn{1}{|c|}{ Timer Duration } \\
\hline \hline T_PRE & $\mathrm{Tr}+\mathrm{Td} 1^{*}+\mathrm{Ts}$ \\
\hline $\mathrm{Tr}$ & $\begin{array}{l}\text { The maximum time to guarantee that UT receives the corresponding } \\
\text { AAL User Data after LT sends ATM primitive (containing 39 octet } \\
\text { AAL User Data). The value of Tr is determined by PIXIT. }\end{array}$ \\
\hline $\mathrm{Td} 1$ & $\begin{array}{l}\text { The maximum processing time of UT to check the data receiving, } \\
\text { generate "Ack" AAL primitive, and send it to IUT. The value of Td1 is } \\
\text { determined by PIXIT. }\end{array}$ \\
\hline Ts & $\begin{array}{l}\text { The maximum time to guarantee that LT receives the corresponding } \\
\text { ATM primitive after UT sent the 39 octet AAL User Data. The value of } \\
\text { Ts is determined by PIXIT. }\end{array}$ \\
\hline
\end{tabular}

* Note : $\mathrm{Td} 1$ may not be included, if $\mathrm{Td} 1$ is significantly smaller than both $\mathrm{Tr}$ and $\mathrm{Ts}$.

\section{2) Sender Mode Timer : T_SEND}

T_SEND timer is used by LT to define the period of time which the LT has to wait to guarantee receiving all ATM primitives that were sent by UT as an AAL User Data from IUT. Tms duration is defined to guarantee that $\mathrm{LT}$ receives corresponding ATM primitives after UT sent AAL User Data. In IUT sender mode testing, UT shall send immediately pre-defined AAL User Data as an AAL_UNITDATA.request primitive after sending "Ack" AAL primitive. Upon receiving the "Ack" 
ATM primitive from IUT, LT starts the T_SEND timer. If the LT receives the last ATM primitives corresponding to AAL_User Data that UT sent, the LT stops the timer T_SEND. When timer T_SEND expires prior to receipt of the last ATM primitive from IUT, the LT stops the test case and assigns a verdict as "FAIL". Figure 4 shows an example of T_SEND timer operation.

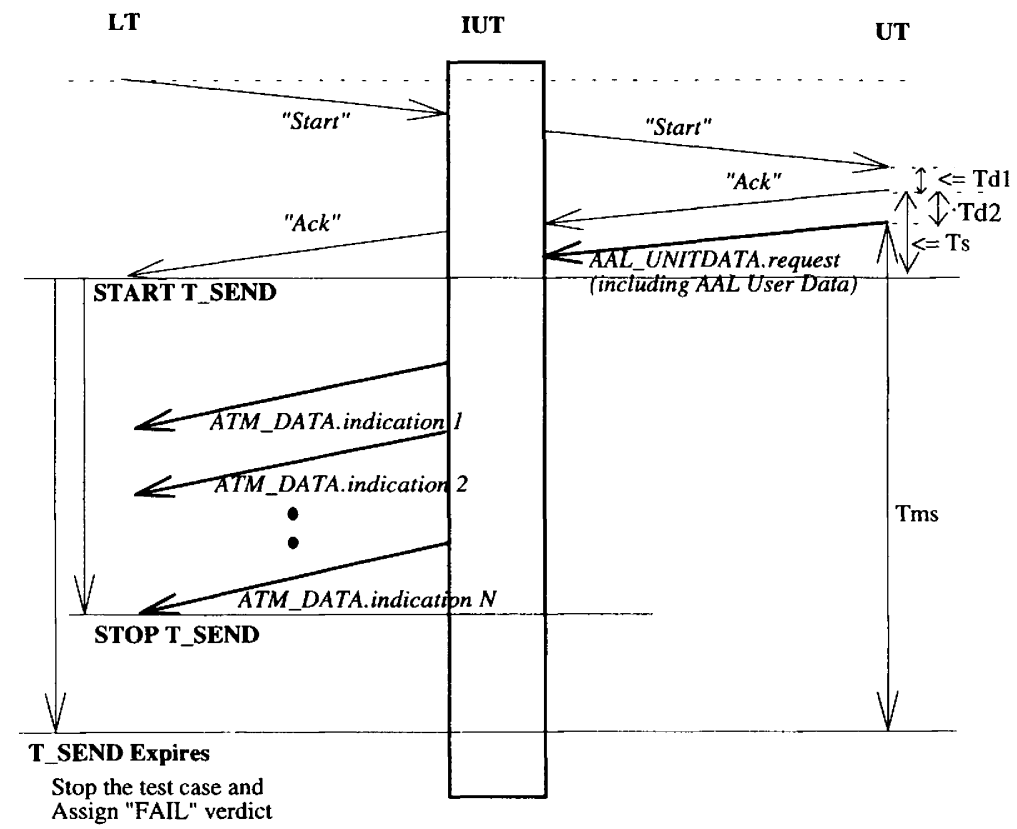

Figure 4 Example of T_SEND timer operation

Table 3 Parameters for sender mode timer operation

\begin{tabular}{|c|l|}
\hline Timer/Parameter Name & \multicolumn{1}{|c|}{ Timer duration } \\
\hline \hline T_SEND & Tms + Td2* - Ts \\
\hline Tms & $\begin{array}{l}\text { The maximum time to guarantee that LT receives corresponding ATM } \\
\text { primitives after UT sent AAL User Data of Max_Deliver_Length size. } \\
\text { The value of Tms is determined by PIXIT. }\end{array}$ \\
\hline Td2 ${ }^{*}$ & $\begin{array}{l}\text { The maximum processing time of UT to generate AAL User Data of } \\
\text { Max_Deliver_Length size and to send it to IUT. The value of Td2* is } \\
\text { determined by PIXIT }\end{array}$ \\
\hline
\end{tabular}

* Note : $\mathrm{Td} 2$ may not be included, if $\mathrm{Td} 2$ is significantly smaller than both $\mathrm{Tms}$ and $\mathrm{Ts}$ separately.

\section{3) Receiver Mode Timer : $T \_$REC}

In IUT receiver mode testing, LT sends "Start" ATM primitive; IUT then sends "Start" AAL primitive 
to UT. Upon receiving the "Start" AAL primitive, the UT sends "Ack" AAL primitive to the IUT and starts T_REC timer. After receiving "Ack" ATM primitive from IUT, the LT shall immediately send the pre-defined ATM primitives which include a pre-defined AAL User Data. If the UT receives AAL User Data corresponding to ATM primitives that the LT sent, the UT stops timer T_REC. When timer T_REC expires prior to receipt of the AAL User Data from the IUT, the UT stops the test case and assigns a verdict as "FAIL". Figure 5 shows an example of T_REC timer operation.

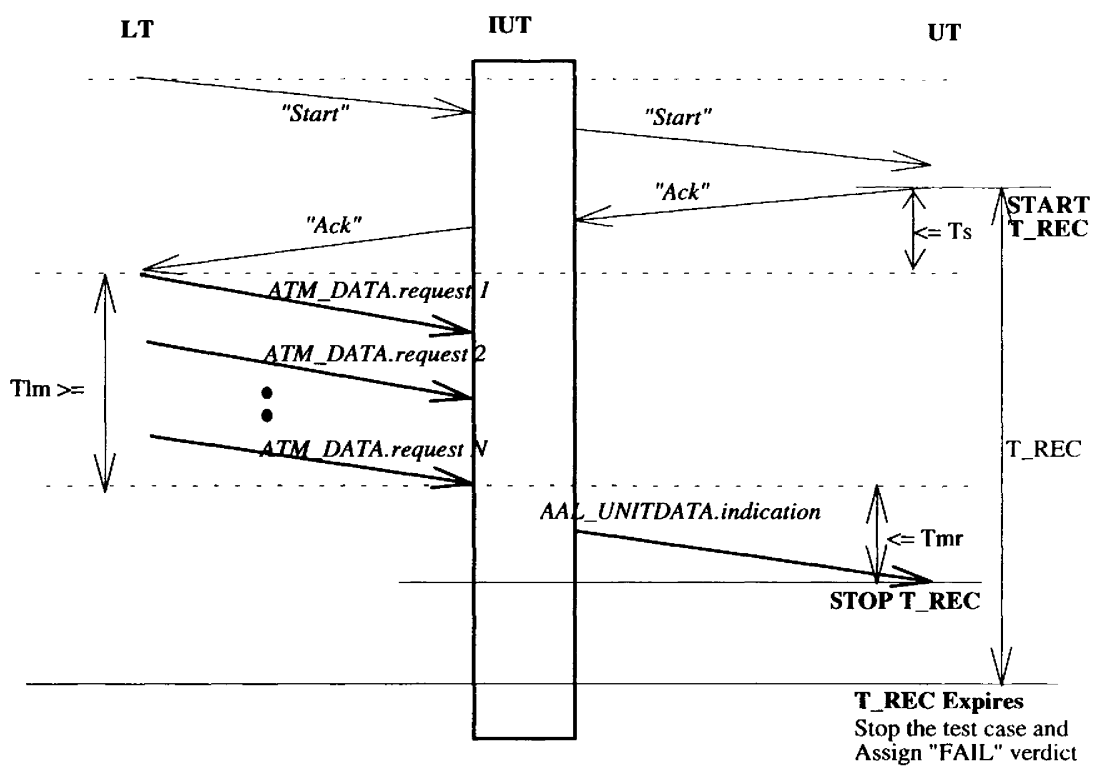

Figure 5 Example of T_REC timer operation

Table 4 Parameters for receiver mode timer operation

\begin{tabular}{|c|l|}
\hline $\begin{array}{c}\text { Timer/Parameter } \\
\text { Name }\end{array}$ & \multicolumn{1}{|c|}{ Timer duration } \\
\hline \hline T_REC & Ts + Tlm + Tmr \\
\hline Tlm & $\begin{array}{l}\text { The maximum time to guarantee that the IUT receives all of the pre-defined } \\
\text { ATM primitives (containing Max_Deliver_Length size AAL User Data) sent by } \\
\text { the LT. The value of Tlm is determined by PIXIT. }\end{array}$ \\
\hline Tmr & $\begin{array}{l}\text { The maximum time to guarantee that UT receives corresponding reassembled } \\
\text { AAL User Data after LT sent pre-defined ATM primitives that contained Max } \\
\text { Deliver_Length size AAL User Data. The value of Tmr is determined by } \\
\text { PIXIT. }\end{array}$ \\
\hline
\end{tabular}




\section{UPPER TESTER FOR CPAAL5 TESTING}

In ISO/IEC 9646 Part 1 (ISO/IEC 9646-1, 1993) and ITU-T Recommendation X.290 (ITU-T X.290, 1992), Upper Tester has been defined as "the means of providing, during test execution, control and observation of the upper boundary of the IUT, as defined by the chosen Abstract Test Method". However, implementing and using the UT have been recognized that it is not easy to apply in real testing environment because direct access to the upper boundary of the IUT is not always supported and coordination between UT and LT requires a complex procedure. Therefore, we propose an UT that has a simple structure. As shown in Figure 6, if UT manager receives "Start" message, then the manager sends "Ack" and calls the indicated test case execution file. According to the test purpose, the UT sends a predefined AAL User Data or waits an expected AAL User Data from the IUT.

The only support expected by UT in SUT is the sending and receiving of AAL User Data and the timer operation management presented in Section 5 . Such minimum capabilities are normally supported by API (Application Programming Interface) which venders provide. Even though some vender's APIs can not handle primitive parameters such as loss priority and congestion indication, recently, standardization bodies including ATM Forum are trying to develop standardized APIs for ATM/BISDN implementation. If the standardized APIs are developed, the UT will be able to be implemented and used more easily.

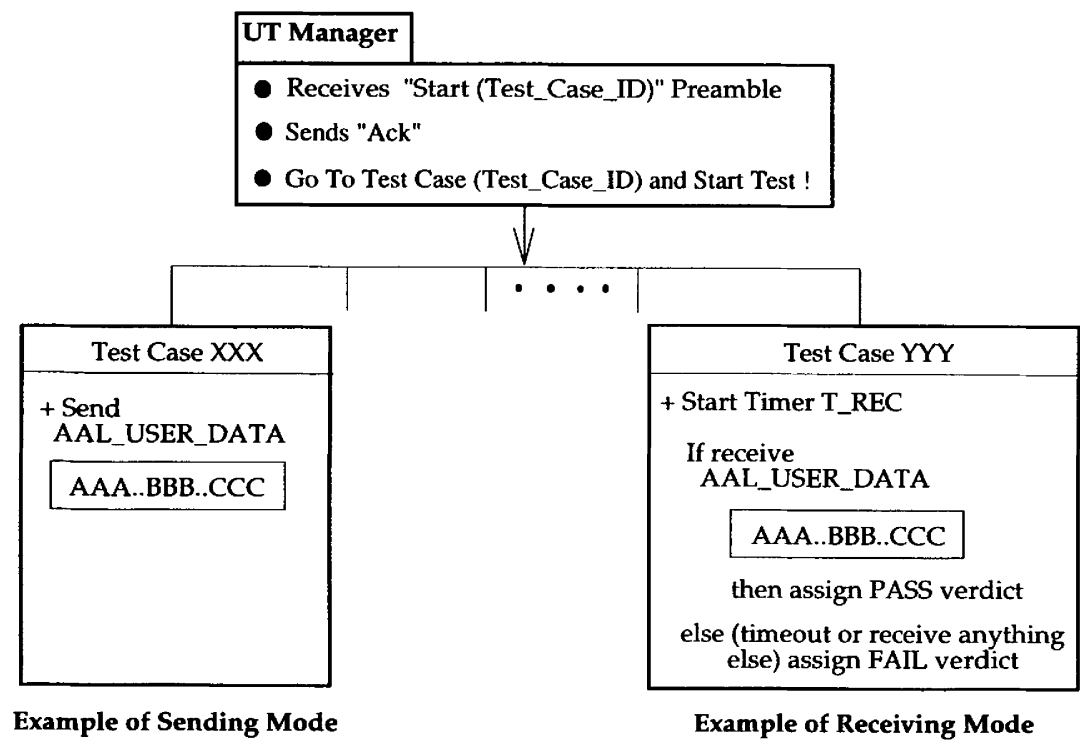

Figure 6 The structure of Upper Tester 


\section{EXAMPLE TEST CASE FOR CPAAL5 TESTING}

An example test case described in TTCN using the proposed test method for CPAAL 5 is given in Table 5. In this test case, the test body calls the test step PREAMBLE presented in Table 6 to initiate the test case execution. In the preamble of Table 6, LT sends "Start" ATM primitive, that contains the test case identification (Test_Case_ID) and the mode flag $(R:=1)$ to indicate receiver mode testing, to IUT. After sending the "Start" ATM primitive, LT starts timer T_PRE. When UT receives "Start" primitive, the UT sends "Ack" primitive to LT and starts timer T_REC. Upon receipt of the "Ack" primitive the LT stops timer T_PRE and starts test body. If timer T_PRE expires prior to receipt of the primitive, the LT stops timer T_PRE and assigns a verdict "INCONCLUSIVE". Since the test case shown in Table 5 is for the IUT's receiver mode testing, the UT expects to receive a corresponding AAL User Data to the ATM primitives sent by the LT. When the UT receives a correct AAL User Data, the UT assigns a "PASS" verdict for the test case. If the UT receives an unexpected AAL User Data or timer T_REC is expired, then a "Fail" verdict is assigned. This test case shows the timing synchronization procedure between UT and LT presented in Section 5.

Table 5 Example of test body for the receiver mode test case

\begin{tabular}{|c|c|c|c|c|c|}
\hline \multicolumn{6}{|c|}{ Test Case Dynamic Behaviour } \\
\hline \multicolumn{6}{|c|}{$\begin{array}{l}\text { Test Case Name : rec_1 } \\
\text { Group : AAL_CP/RECEIVER/ } \\
\text { Objective : Verify that the IUT recovers and delivers the CPCS-SDU to the upper layer upon reception of } \\
\quad \text { an ATM-SDU with the AUU parameter in ATM_DATA.indication primitive equal to } 1 . \\
\text { Configuration : } \\
\text { Default : }\end{array}$} \\
\hline $\mathbf{N r}$ & $\mathbf{L}$ & Behaviour Description & Constraints Ref & $\mathbf{v}$ & Comments \\
\hline $\begin{array}{l}2 \\
3 \\
4 \\
5 \\
6 \\
7 \\
8\end{array}$ & & $\begin{array}{l}\text { (Test_Case_ID:="rec_1",R:=1) } \\
\text { +PREAMBLE(Test_Case_ID,R) } \\
\text { LT_PCO!ATM_D.req } \\
\text { LT_PCO!ATM_D.req } \\
\text { LT_PCO!ATM_D.req } \\
\text { UT_PCO?AAL_UD.ind } \\
\text { UT_PCO?OTHERWISE } \\
\text { ?TIMEOUT T_REC }\end{array}$ & $\begin{array}{l}\text { ATM_D_R_1_1 } \\
\text { ATM_D_R_1_2 } \\
\text { ATM_D_R_1_3 } \\
\text { AAL_UD_I_1 }\end{array}$ & $\begin{array}{l}\mathbf{P} \\
\mathbf{F} \\
\mathbf{F}\end{array}$ & 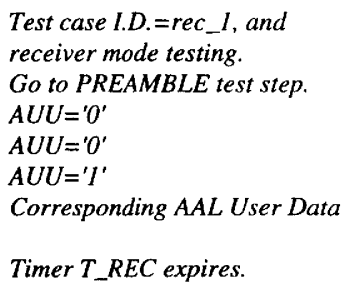 \\
\hline
\end{tabular}


Table 6 Example of preamble

\begin{tabular}{|c|c|c|c|c|c|}
\hline \multicolumn{6}{|c|}{ Test Step Dynamic Behaviour } \\
\hline \multicolumn{6}{|c|}{$\begin{array}{ll}\text { Test Step Name } & : \text { PREAMBLE(Test_Case_ID, R) } \\
\text { Group } & : \text { AAL_CP/ } \\
\text { Objective } & : \text { Procedure used to notify the start of the test case to the Upper Tester. } \\
\text { Default } & : \\
\end{array}$} \\
\hline $\mathbf{N r}$ & $\mathbf{L}$ & Behaviour Description & Constraints Ref & $\mathbf{v}$ & Comments \\
\hline 1 & & LT_PCO!ATM_D_req & $\begin{array}{l}\text { ATM_PRE_Start } \\
\text { (Test_Case_ID,R) }\end{array}$ & & LT sends "Start" to the IUT. \\
\hline 2 & & START T_PRE & & & \\
\hline 3 & & UT_PCO?AAL_UD_ind & $\begin{array}{l}\text { AAL_PRE_Start } \\
\text { (Test_Case_ID,R) }\end{array}$ & & UT receives "Start" from the IUT. \\
\hline 4 & & UT_PCO!AAL_UD_req & AAL_PRE_Ack & & UT sends "Ack" to the IUT. \\
\hline 5 & & {$[R=1]$} & & & (Receiver mode testing) \\
\hline 6 & & START T_REC & & & Timer $T_{-} R E C$ is started by $U T$. \\
\hline 7 & & +LT_PRE & & & \\
\hline 8 & & {$[R=0]$} & & & (Sender mode testing) \\
\hline 9 & & +LT_PRE & & & \\
\hline 10 & & UT_PCO?OTHERWISE & & I & \\
\hline 11 & & ?TIMEOUT T_PRE & & I & Timer T_PRE expires. \\
\hline 12 & & LT_PRE & & & \\
\hline 13 & & LT_PCO?ATM_D_ind & ATM_PRE_Ack & & LT receives "Ack" from the IUT. \\
\hline 14 & & LT_PCO?OTHERWISE & & I & \\
\hline 15 & & ?TIMEOUT T_PRE & & I & Timer $T_{-} P R E$ expires. \\
\hline
\end{tabular}

\section{CONCLUSION}

In this paper, we analyzed test requirements for AAL Type 5 protocol conformance testing. To choose the best test method, we compared test coverage of four applicable test methods, i.e., Local Test Method, Remote Test Method, Distributed Test Method, and Coordinated Test Method after analyzing the restrictions of AAL Type 5 protocol with respect to conformance testing. We found that the Distributed Test Method is the most efficient method in the view point of test coverage and real test environments. To provide necessary timing synchronization and test coordination between LT and UT, we proposed a simple mechanism that uses only three timers. We developed an abstract test suite for AAL Type 5 Common Part protocol testing using proposed mechanism. Finally, we presented an example of test case to show how we described behaviour of the test specification using TTCN.

This paper shows a part of our ongoing research in broadband network protocol testing project. Currently, we are developing test suites for AAL and signalling protocols to prepare testings for broadband network services and to contribute to ATM Forum testing specification works. For further research, we have plans to implement an UT using standardized API and to generate test cases using SDL automatically.

\section{REFERENCES}

Ana R, Cavalli, Jean-Philippe Favreau, and Marc Phalippou (1994), "Formal Methods in Conformance 
Testing: Results and Perspectives", Protocol Test Systems, VI.

G. Gonenc (1970), "A Method for the Design of Fault-Detection Experiments", IEEE Trans. Comput., vol. 19.

ISO/IEC 9646-1 (1993), "Information Technology - Open Systems Interconnection - Conformance Testing Methodology and Framework - Part 1 : General Concepts".

ISO/IEC 9646-3 (1993), "Information Technology-Open Systems Interconnection-Conformance Testing Methodology and Framework-Part 3:Tree and Tabular Combined Notation (TTCN)".

ITU-T COM SVIII-R 116 (1992), "B-ISDN ATM Adaptation Layer (AAL) functional description".

ITU-T TD-XVIII/10 (1993), "AAL Type 5, Draft Recommendation text for section 6 of I.363".

ITU-T X.290 (1992), "Information Processing System - OSI Conformance Testing Methodology and Framework - Part 1 : General Concepts".

Krishan Sabnani and Anton Dahbura (1988), “A Protocol Test Generation Procedure”, Computer Networks and ISDN Systems, vol. 15.

Marc F. Witteman and Ronald C. van Wuijtswinkel (1993), "ATM broadband network testing using the ferry principle", Protocol Test Systems, V.

Nouni (1995), "Test Purposes for the AAL Type 5 Common Part Conformance Testing", ATM Forum 95-0033.

Sangjo Yoo and Leslie Collica (1995), "Timing Synchronization for the CPAAL5 Conformance Testing using Distributed Test Method", ATM Forum 95-0309, April 1995.

Tsun S. Chow (1978), “Testing Software Design Modeled by Finite-State Machines", IEEE Trans. on Software Engineering, vol. SE-4.

Zeng H.X, Chanson S.T., and Smith B.R. (1989), "On Ferry Clip approaches in protocol testing", Computer Networks and ISDN Systems 17.

\section{BIOGRAPHY}

Sangjo Yoo received BA in electronic communication engineering from Hanyang Univ. in 1988, MS in electrical engineering from the Korea Advanced Institute of Science and Technology in 1990. Currently he is with the Korea Telecom Research and Development Group as a member of technical staff and serving as an editor in the ATM Forum. His research interests include protocol testing and formal description technique.

Leslie A. Collica is a Computer Scientist working at the National Institute of Standards and Technology since 1988 on ISDN and Broadband ISDN protocols, conformance and interoperability tests. She is Editor of the Test Working Group in the ATM Forum and is Chairman of the North American ISDN User's Forum. She has a B.S. degree from the University of Maryland.

Myungchul Kim received BA in electronics engineering from Ajou Univ. in 1982, MS in computer science from the Korea Advanced Institute of Science and Technology in 1984, and Ph.D in computer science from the Univ. of British Columbia in 1992. Currently he is with the Korea Telecom Research and Development Group as a managing director, Chairman of Profile Test Specification-Special Interest Group of Asia-Oceania Workshop, and will be Chair of the 10th IWTCS'97. His research interests include protocol engineering on telecommunications and multimedia. 